\title{
ماهية الدلالة المعجمية ومراحلها
}

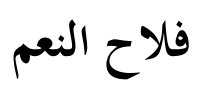

\section{مدرس قسم تدريس اللغة العربية في الجامعة الإسلامية الحكومية بنجكولو}

\author{
(البريد الإلكتروين: falahun@iainbengkulu.ac.id)
}

\begin{abstract}
Abstact
Dictionaries, a book that includes the largest number of vocabulary combined with explaining and interpreting their meaning, to be material arranged a special arrangement: either the letters of the alphabet, or on the subject. It is called a dictionary because the nouns remove the confusion and clarify the vagueness. The complete of dictionary, which includes every word in accompanied by explaining its mean and its derivation and the method of its pronunciation and evidence showing its language positions used.

Lexicology is the part of linguistics that interest with the study of words, its nature and its mean, the elements of words, the relations between words (semantic relationships), the sets of words and the study of each lexicon of a language. The linguists divided the stages of language collection into several stages, each stage different from one another, everything will be discussed in this article.
\end{abstract}

Keyword: Significance, Lexicon, Stages 
مرتبـة ترتيبـاً خاصـا : إمـا على حروف

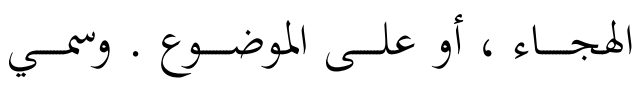

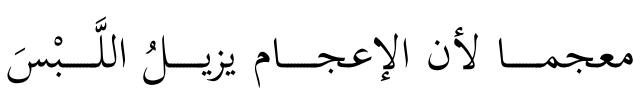

$$
\text { ويُوضح المبهم . }
$$

والمعجم الكامل هو الذي يضم كل كلمة في اللغة مصحوبة بشرح معناها واشتقاقها وطريقة نطقها وشواهد تبين مواضع استعمالها ـ عرفنا فيما سبق أن الرموز ما أن تكون لغوية أو اصطلاحيا، وأن علم الدلالة يهتم بالرموز بأنه رمز قابل للتحليل، ذلك لان طبيعية نطقية وطبيعية فيزيائية وطبيعية سمعية في مستوى الصوتى والصرفي والتركيب. ويتنوع علم الدلالة إلى عليها المختلفة منها الدلالة الصوتية، الدلالة الصرفية، الدلالة النحوية، الدلالة المعجمية والدلالة السياقية. وقد شرحها مسبقا المجموعة القادمة عن تعاريف جميع فروع علم الجملالة، ونخن كمجموعة الثالثة سنجرّب عن بيان ماهية الدلالة
إن علم المعاجم كعلوم أخرى يتطور ويتبسـط مبحثـه حسـب مـرور الـزمن، وذلك لا لشيئ إلا لأجل خدمة كتاب الله وسـنة رسـوله صـلى الله عليـه وسـلم

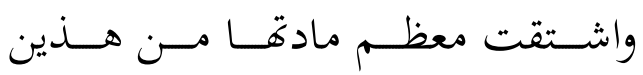
المصـدرين الجليلـين، فكانـت في بـدايتها تفسيرا لبعض مـا غمض على الصحابة من القرآن والسنة,وكان سيدنا ابن رضي

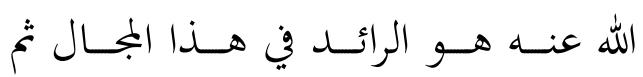
تطورت هذه الحركة بعد اخطلات العرب بغـيرهم وانتشـار اللحـن علـى الأســة فـذهب العلمـاء إلى الباديـة وجمعـوا مــا جمعوا مـن ألفـاظ صـنفوها في تصـانيف كثـيرة متعـددة اختلفــت في مناهجهـا وترتيبها ونظامها ومسن ثم فقـد اختلفـت

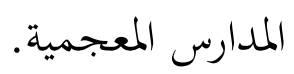
المعاجم وهو كتاب يضم أكبرَ عدد مـن المفردات اللغويـة مقرونـة بشـرحها ، وتفسير معانيها ، على أن تكون المواد 
المعحمية ومراحلها، عسى أن يكون هذا اقذيت عين الصبي : ازلت ما بها من البحث مدخلات حسنة لزيادة معارف ق قلذى 1.

وعلى هذا يصير معنى أعجم : أزال لنا في علم الدلالة ويمكن أن نألف أو العجمة أو الغموض أو الإجهام . ومن ندرّن المعجم المخصّ لنا. هنا أطلق على نقط الحروف لفظ "الإعجام" لأنه يزيل ما يكتنفها من غموض أو الإهمام. فمثلا حرف "ب" أ- تعريف المعجم لغة

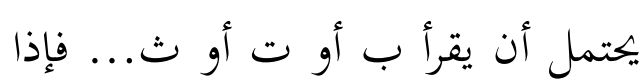
وضعنا النقط أى أعجمناه زال هذا الإحتمال وارتفع الغموض 2. ب- - تعريف المعجم اصطلاحا فتعريف المعاجم اصطلاحا فهو عند علماء اللغة كتاب يضم ألفاظ اللغة مرتبة على نمط معين مشروحة شرحا يزيل إبهامها، ومضافا إليها ما ينا سبها

1. احمد مختار عمر , صناعة المعجم الحديث، القاهرة: عالم الكتب, 1998 2. نفس المرجع, ص. 19 ص. 19 تفيد مادة "عجم" في اللغة معنى الابهام و والغموض، فقى اللسان: الأعجم الذي لا يفصح ولا يبين كلامه، وفيه رجل أعجمي وأعجم : اذا كان في لسانه عجمة، وفيه سميت البهيمة عجماء لأها لا تتكلم. وسمى العرب بلاد فارس بلاد العجم لأن لغتها لم تكن واضحة ولا مفهومة عندهم. فإذا ادخلنا الهمزة على الفعل "عجم" ليصير "أعجم" اكتسب الفعل معنى جديدا من معنى الهمزة (او الصيغة) الذي يفيد هنا السلب والنفي والإ زالة. ففي اللغة أشكيت فلانا : أزلت شكايته، وفيها 
وهي الدلالة التي نوجه إليها هنا كل

عنايتنا، كالدلالة التي تستفاد من "التصديق" ودلالة "الكذب" و"النفط" و "النصوخ" الخ.. فكل كلمة من كلمات اللغة لها دلالة معجمية او اجتماعية تستقل عما يمكن ان توجيه اصوات هذه الكلمة او صيغتها من دلالات زائدة على تلك الدلالة الأساسية التي يطلق عليها الدلالة الإجتماعية4. وتسمى أيضا بالدلالة الأساسية للكلمة، فكل كلمة من الكلمات لها معني أساسي تواضع عليه الجماعة وصار عندهم عرفا، وقد يطرأ على معناها بعض التغيير إذا ركبت في جملة فيكون للسياق دور في اكتساها معني طارئا، أو يؤدي المقام إلى استحداث معني لهذه الكلمة، أو يقوم المجاز بهذا الدور فيكسبها معني جديدا، إلا أن الكلمة في جميع الأحوال تظل محتفة بالمعني

4 .ابرهيم انيس ,دلالة الألفاظ ,القاهرة : الأنجلو المصرية,1991, ص.48.
من المعلومات التي تفيد الباحث وتعين الدارس على الوصول إلى مرادهُ3. وأما علم المعاجم (Lexicology) هو ذلك الجزء من علم اللسانيات الذي يهتم بلدراسة الكلمات وطبيعتها ومعناها، وعناصر الكلمات، والعلاقات بين الكلمات (العلاقات الدلالية)، وبجموعات الكلمات و و دراسة كل المعجم للغة من اللغات. ويتربط علم المعاجم بعلم آخر هو علم صناعة المعاجم (Lexicography) ، ومن أشهر المعاصرين العرب الذين لهم دراسات جادة ومفيدة في علم المعاجم العالم العراقي علي القاسمي. كما أن مكتب تنسيق التعريب بالرباط وبجلته اللسان العربي لمما اهتمام كبير بعلم المعاجم وعلم صناعة المعاجم. ج- تعريف الدلالة المعجمية

3 .احمد علي محمود ربيع, المدخل الى علم المعاجم والدلالة، الرياض:

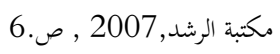


الأساسي لها ولا تفرط فيه مع قبولها

د-وظائف المعجم وموقف المعجم

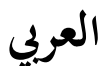

أما وظائف المعجم فكما يلي:

أ. شح معاني المفردات

بوضعها في سياقات متعددة

ب.بيان ضبط الكلمة بالشكل

ت.بيان كيفية كتابة الكلمة في

حالة اختلاف النطق عن

الكتابة كما في : الله-

الرحمن - السموات- هذا

- لكن.

ث.دراسة الصوات

باعتبارها عاملا مساعدا

على توضيح المعنى. فاذا

نظرنا إلى المعاجم العربية

وجدنا أها تنقسم إلى

قسمين حسب اهتمامها

بدراسة أصوات اللغة.
لهذه المعاني المكتسبة، ومن ثم يتميز

معناها الأصلي بأنه المعني الأساسي.5

يصطلح علم المعاجم في كل لغة

بالكشف عن الدلالة المعجمية للكلمة "فدراسة المعنى المعجمي تشكل قطاعا عريضا وأساسيا من علم المعاجم Lexicology المعاجم أن دراسة المعنى المعجمى هو الهدف الأول لهذا العلم ودراسة المعنى المعجمى تعتبر أول خطوة للحديث عن الكلمة ودلالتها، ذلك لأن الدلالة الصوتية و والصرفية و والنحوية، تعتبر دلالات وظيفة ويطلق عليها الدكتور تمام حسان مصطلح "المعنى الوظيفي لأن لكل واحد من هذه الأمور (يقصد الصوت و والحرف والموقع والصيغة والباب) وظيفة خاصة يؤديها، ويساهم بأدائها في بيان المعنى العام ووضوحه.

5 أحمد علي محمود ربيع، المدخل إلى علم المعاجم والدلالة، (الرياض : مكتبة الرشد، 2007 م)، ص. 121-122. 
د. التنبيه برموز معيّنة على

الفصيح والمعرّب و والدّخيل

والمولّد

ذ. اشتماله على مصطلحات

$$
\text { العلوم والفنون }
$$

ر. ذكر الشواهد عند الضرورة

لتوضيح المعنى 6

وقد حصر المعجميون أهم

وظائف المعجم فيما يأتي :

$$
\text { أ. ذوسر المعنى }
$$

ب.بيان النطق (ويدخل فيه

التقسيم المقطعي وموضع

$$
\text { النبر) }
$$

$$
\text { ت.تحديد الرسم الإملائي }
$$

$$
\text { ث. التأصيل الاشتقاقي }
$$

ج. المعلومات الصرفية والنحوية

$$
\text { ح. معلومات الاستعمال }
$$$$
\text { خ. المعلومات الموسوعية }
$$

القسم الأول يهتم بدراسة

أصوات اللغة، ويمثله لسان

العرب لابن منظور والمعجم

الوسيط الذي قامت بعمله

لجنة من المجمع اللغوي

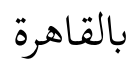

القسم الثاني لا يهتم بدراسة

أصوات اللغة، ويمثله أساس

البلاغة للزحشري والصحاح

للجوهري والقاموس المخيط

$$
\text { لميروز ابدي. }
$$

ج. تقديم معلومات نحوية

أساسية مثل : تعدي الفعل

$$
\text { ولزومه. }
$$

ح. تقديم معلومات صرفية

أساسية عن الكلمة

$$
\text { (نوعها-تصريفاتما) }
$$

خ. تقديم الصور لكل ما يمتاج

شرحه إلى ذلك

6 6مر سليمان محمد و الطيب سوسي,دليل الطالب في استخدام

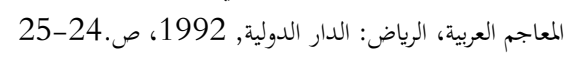


المالح، وهذا هو القدر المشترك من الدلالة، بين أفراد المجتمع لهذه الكلمة. 2. ما تتضمنه الكلمة من دلالات،

أو ما تستدعيه في الذهن من

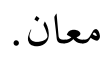

كما يطلق مصطلح الدلالة الهامشية

على العنصر الثاني، ويعنى بها تلك الظلال من المعانى التي تختلف من فرد إلى آخر، تبعا لتجارب الأفراد و و خبراهم، وما ورثه عن آبائهم وأجدادهم. أما الدلالة الهامشية فهي تختلف من فرد إلى آخر، حسب المخزون من خبراته ونجاربه الخاصة، فالأولى دلالة عامة ومشتركة، والثانية دلالة خاصة وفردية، إذت تمثل "جميع العناصر الدلالية التي ليست لها صلة مباشرة بما تشير إليه الكلمة في الخارج".

3. درجة التطابق بين العنصر الأول والثاني.

\section{هـ - عناصر الدلالة المعجمية}

ويرى علماء اللغة المحدثون

والمعاصرون، وفي مقدمتهم علماء المعاجم أن المعنى المعجمى Lixial يتكون من عناصر رئيسية Meaning 7. ثلاثة 1. ما تشير إليه الكلمة في العالم

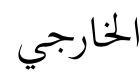

ويطلق الدكتور إبراهيم أنيس مصطلح الدلالة المركزية، على العنصر الأول، والمراد بالدلالة المركزية، ذلك القدر المشترك من الدلالة، الذي يعرفه أفراد المجتمع للكلمة، والذي نصل كمم إلى فهم هذه الكلمة، وقد تكون هذه الدلالة المركزية واضحة في أذهان كل أفراد المجتمع، كما قد تكون مبهمة في أذهن بعضهم. فلكلمة البحر مثلا لها دلالة مركزية، على ذلك المجرى الواسع الملئ بالماء

7 فريد عوض حيدر، علم الدلالة دراسة نظرية وتطبيقية، ( قاهرة :

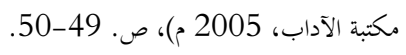




$$
\text { أما دراجة التطابق بين دلالتين 1- عام }
$$

فللكلمة معنى عام معجم، ذلك

لأها ليست في سياق محدد إذا السياق

هو الذي يحدد هذا المعنى العام ويقيده.

المثال "نظر"، وتأتي منه معانى ، يرى

شيئا، يشاهد شيئا، يطلّ وغيرها.

$$
\text { 2-2 - متعدد }
$$

أما كون المعنى الكلمة متعدد في

المعجم ذلك لأغا تصلح للدخول في

سياقات متعددة، فيعطها كل سياق

معنى، ومن إستخدامها في النصوص

العربية القديمة والحديثة تكتسب هذ

التعدد. المثال "رأس"، و تأتي منه معانى :

رأس الناس، رأس من المدرسة (رئيس

المدرسة، رأس بمعنى نفس (لكل رأس رس رست

واجبات)

3- غير الثابت

وهو معنى غير الثابت لأن دلالة الكلمة تتعرض لتغيير فيصيبها التعميم

\section{و- خصائص الدلالة المعجمية}

يحدد المحدثون من اللغويين ثلاثة

المركزية و الهامشية فقد أشار إليها

زجوستا وهو يعني بهذه الدلالة مدى التطابق بين هاتين الدلالتين فإذا أخذنا كلمة الماهية وكلمة الأجرة على سبيل المثال وجدنا أن بينهما تطابقا في الدلالة المركزية لأهما تشتركان في الدلالة على ما يأخذ المرء من المال نظير عمل يقوم به، ولكن بينهما فرقا يتمثل في درجة التطابق بينهما، فالماهية تدل على ما تأخذه طبقة من الموظفين كل شهر، أما كلمة الأجرة فهي تدل على مبلغ الذي تتسلمه طبقة أخرى كل يوم نظير عمل يوم. خصائص للمعنى المعجمي، تعد من أبراز قصائص هذا المعنى تلك هي أنه : 
زيد وثعلب وأبي علي القالي

وغيرهم.

3. جمع الألفاظ الخة

بموضوع واحد وذلك ككتب

الخيل، و والنبات، و والإبل،

والعصا، وخلق الإنسان

وغيرها من الرسائل الصغيرة

التي ت تحتوى على ألفاظ

$$
\text { الموضوع الواحد. }
$$

4. جمع الألفاظ الغريبة مع

شرحها وتفسيرها

والاستشهاد عليها من كلام

العرب شعرهم ونثرهم

وأمثالهم، من ذلك كتب

غريب اللغة والشوارد، ومنها

أيضا غريب القرآن وغريب

الحديث وقد تناوله بجموعة

$$
\text { كبيرة من العلماء. }
$$

5. جمع الألفاظ الموضوعة

لمختلفة المعاني في كتاب
أوالتخصيص أو الإنتقال. المثال "عربة"،

في الزمن السابق معناها "سيارة" والأن

تكون آلة لحملة شيئ.

ز- - مراحل جمع اللغة

قسم العلماء جمع اللغة والتعامل

هذا الجمع إلى مراحل متعددة تختلف

كل مرحلة منها عن الأخرى من

حيث المنهج والتناول. وأكمل هذه

المراحل من ناحية الفكر المعجم في

تناول المادة وشرحها وتنظيمها

وترتيبها. والمراحل التي مربها الجمع

ماهي :

1. مرحلة جمع بطريقة عشوائية

2. مرحلة جمع الألفاظ مع لع

الذكر القصص والأحاديث

والنوادر والغرائب التي وردت

فيها هذه لألفاظ، تسمى

بكتب النوادر كنوادر أبي 
أن الدلالة المعجمية هي كل

كلمة من الكلمات التي لها أسس

وتواضع عليه الجماعة وصار عندهم عرفا

أي لكل مقصود خاص مباينا لاعترافه إلى مفهوم جديد.

زنرى بعض عناصر الدلالة

المعجمية من بعض اللغويين ونستنبطها

أن في الدلالة المعجمية عناصر معينة

منها : ما تشير إليه الكلمة في العالم

الخارجي، وعنصر الثانى ما تتضمنه

الكلمة من دلالات، أو ما تستدعيه في

الذهن من معان، والثالث التطابق

بينهما. إذا لسهولة فهم أو تعيين المعنى

المعجمى أن تكون فيه تلك العناصر

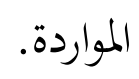

ولتدوين المعجم لابد أن يشترك المألف

بعض مراحل التي تتعاون إليه منها :

مرحلة جمع بطريقة عشوائية، مرحلة جمع

الألفاظ مع الذكر القصص والأحاديث والنوادر والغرائب التي وردت فيها هذه
واحد، وتسمى معاجم

المعاني و والموضوعات، وهي

متعددة منها الغريب

المصنف لأبي عبيد، ومبادئ

اللغة للإسكافي، وفقه اللغة

للثعلبي، والمخصص لابن

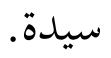

جمع الألفاظ بطريقة حاصرة مع شرحها

وتبوبيهاحسب منهج معين، وهذه

تسمى بالمعاجم العامة أو المجنسة ومنها

العين للخليل بن أحمد، والتهذيب

للأزهري والصحاح للجوهري وغيرها.

الحنالاصة

علم المعاجم (Lexicology) هو

ذلك الجزء من علم اللسانيات الذي

يهتم بدراسة الكلمات وطبيعتها ومعناها، وعناصر الكلمات، والعلاقات بين الكلمات (العلاقات الدلالية)، وبجموعات الكلمات و دراسة كل المعجم للغة من اللغات. 
لألفاظ، جمع الألفاظ الخاصة بموضوع أحمد علي محمود ربيع، المدلخل إلى علم

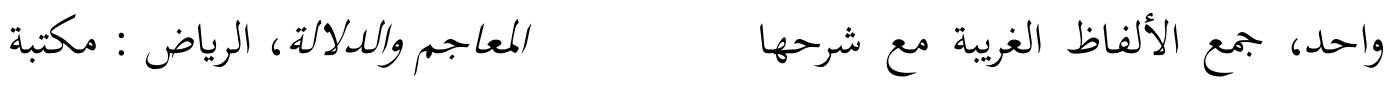
وتفسيرها والاستشهاد عليها من كلام الرشد، العرب شعرهم ونثرهم وأمثالم، جمع فريد عوض حيدر، علم الدلالة دراسة نظرية وتطبيقية، قاهرة : مكتبة الألفاظ الموضوعة لمختلفة المعاني في الآداب، 2005 م كتاب واحد، جمع الألفاظ بطريقة احمد غختار عمر , صناعة المعجم حاصرة مع شرحها وتبوبيهاحسب منهج الحديث، القاهرة: عالم الكتب,

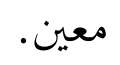

\section{8}

ابرهيم انيس ,دلالة الألفاظ ,القاهرة :

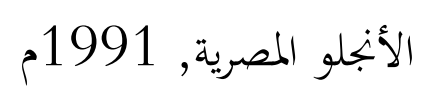

المراجع 